\title{
Equalizer Design Based on ATSC-M/H Protocol
}

\author{
T. Xu, Z.H. Hu, Z.F. Wang, H.H. Song \\ Shanghai Second Polytechnic University \\ Shanghai, China
}

\begin{abstract}
This paper analyzes the channel model under mobile reception state firstly, then study the self-correlation characteristic of ATSC-Mobile DTV training sequence. According with the mobile channel characteristic and selfcorrelation of training sequence, proposed a parallel channel estimation algorithm with multi-channel to reduce the channel estimation time and to improve the estimation efficiency. Meanwhile, proposed a DFE equalizer structures based on sparse algorithm and validated the performance by simulation.
\end{abstract}

Keywords-ATSC-M/H; channel estimation; sparse algorithm; equalizer

\section{INTRODUCTION}

Equalizer is the key technology in Digital TV reception technology, it directly related to the performance of the receiver. The main work of this paper is combined with the ATSC-M/H protocol and digital television transmission characteristics to study how to improve the performance of the equalizer $[1,2]$.

The wireless transmission system's performance is restricted by the channel. And the signal transmission between the transmitter and receiver is very complex, Doppler frequency caused by the relative motion between the receiver and transmitter will influence the receive quality.

Mobile digital TV broadcasting in VHF/UHF frequency band, with the influence of various interference discussed before, which makes the ISI very serious and make the received data cannot meet the needs of information broadcast requires, so the equalizer is needed to eliminate inter-channel interference. The tap values of equalizer is a basic factor that affecting the receive effects, at the same time, in order to improve the user experience degrees, quick initialization equalizer is another key technical on measure the mobile TV reception [3].

Tap initialization algorithm based on training sequences and blind equalization algorithm are most commonly used equalizer initialization algorithm. There are two algorithm to initialize the equalizer taps that based on training sequences. one use the training sequence to estimate the channel impulse response, another one directly use the training sequence to initialize the equalizer[4]. In order to accelerate the equalizer convergence, this paper uses the channel estimation technique to quick initialize the equalizer, the algorithm makes the equalizer open eye quickly and then tracking the channel change by decision oriented algorithm.

\section{SOLUTION TECHNOLOGY}

\section{A. Under the Mobile Reception of Channel Model Analysis}

Training sequence was inserted into the data stream of modern mobile digital television terrestrial broadcasting system, in order to improve the receiver's signal error correction ability and fast tracking ability. The ATSC-M/H standard discussed in this article inserted several training sequence in it's data flow to against channel fading that caused by multi-path channel interference and Doppler frequency shift, which caused the inter-symbol interference. The ATSC-M/H standard is backward compatibility with traditional ATSC standard, so we study the digital TV signals transmission characteristics in Doppler model and static channel model respectively, and carry on simulation analyze to study the effect of channel estimation algorithms to find out which equalizer algorithm is the much better equalize scheme.

ITU channel model is usually used to simulate the mobile receiving channel [5], the channel model includes three test environments: indoor, outdoor to indoor or pedestrian, vehicle movement or high antenna. Meanwhile, for the propagation delay obvious variable, each test environment can be divided into two modes. And total number is six.

To represent the static channel model of receiving, in this article we uses the Brazil channel model for simulation analysis, the model is commonly used for simulate the ATSC standard state channel [6].

\section{B. Autocorrelation Research on Training Sequence}

The ATSC-M/H standard's data frame structure is compatible with the traditional ATSC 8-VSB data transmission structure, each 313 data segment composed a field synchronization based on PN training sequence, each segment structure with four sections synchronization symbol, 700 symbols and training sequence related to the frame of information data of 832 symbols[7]. The 700 training sequence symbols are ${ }^{[\mathrm{PN511}}$ PN63, \pm PN63, PN63].

Therefore, in this section, we consider the ATSC Mobile DTV frame peculiar training sequence, also want to consider traditional based on PN training sequence to estimate the channel quickly. PN sequence is usually generated by feedback shift register. And feedback shift register can be divided into linear and nonlinear, both of which is the longest period of data sequences, ATSC Mobile DTV standard in PN training sequence is generated by using this method $[8,9,10]$.

The normalized autocorrelation function $R_{x}(\tau)$ of signal $x(t)_{\text {with }} T_{0}$ period can be expressed as follows 


$$
\begin{aligned}
R_{x}(\tau) & =\frac{1}{K}\left(\frac{1}{T_{0}}\right) \int_{-T_{0} / 2}^{T_{0} / 2} x(t) x(t+\tau) d t \quad-\infty<\tau<\infty \\
K & =\frac{1}{T_{0}} \int_{-T_{0} / 2}^{T_{0} / 2} x^{2} d t
\end{aligned}
$$

PN511 Self-correlation results can show clearly the characteristics of self-correlation in figure 1.

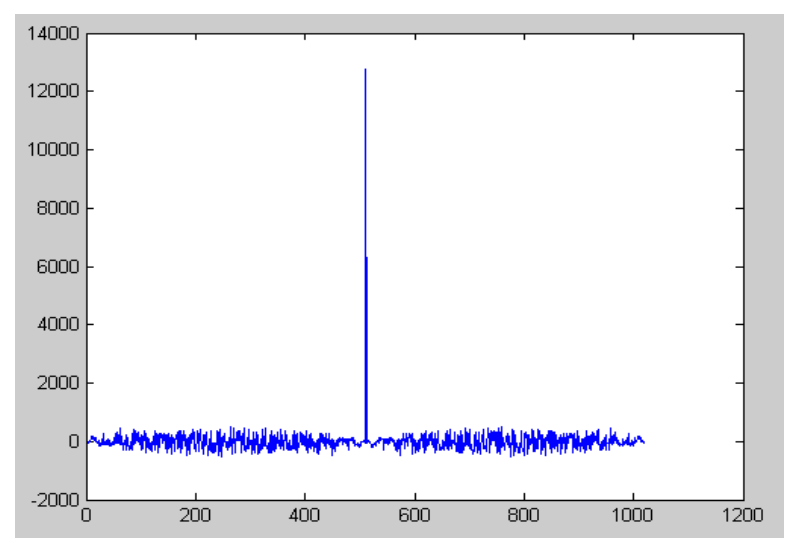

FIGURE I. SIMULATION OF PN511 SELF-CORRELATION.

\section{Parallel Channel Estimation with Multiple Training Sequence Structure}

Traditional ATSC receiver training sequences often uses a single training sequence to estimate the channel and equalize the processor. According to the characteristics of training sequences, we proposed a fast equalizer initialization scheme with parallel structure which based on channel estimation. The structure of channel estimation module not only can quickly synchronization data by different training sequences, but also can be used to estimate the channel pulse fast, make the equalizer fast tracking channel changes and convergence effectively even in bad channel conditions. Compared with previous fixed receive channel equalizer, the algorithm structure can adaptive any kind of training sequence in ATSC$\mathrm{M} / \mathrm{H}$ standard, and can quickly detect the training sequence synchronization and initialize the equalizer tap coefficients in a relative short interval. The parallel structure of fast channel estimation is shown in figure 2 below.

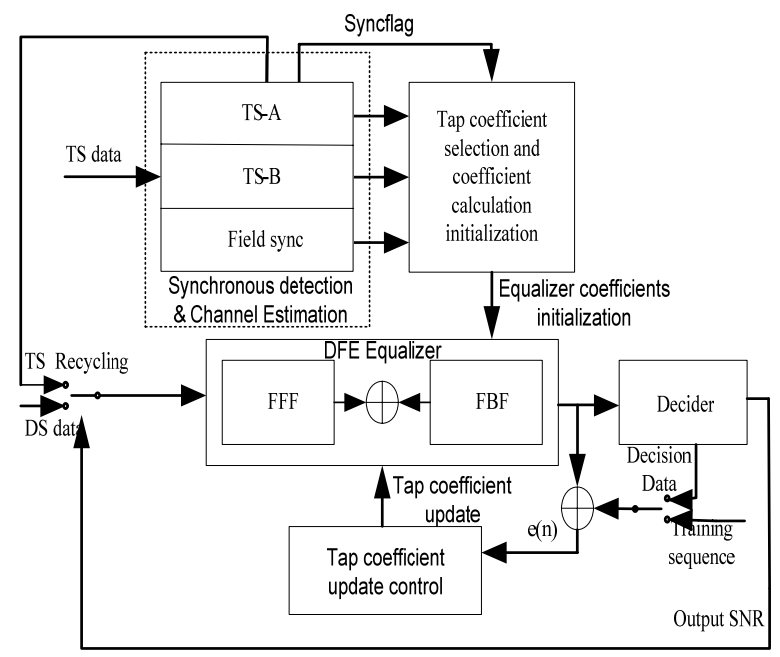

FIGURE II. PROPOSED FAST CHANNEL ESTIMATION STRUCTURE.

The received data transmitted to the synchronous detection module and channel estimation module respectively, calculate with TS-A, TS-B and Filed sync respectively to detect synchronous signal. Each parallel module in the dotted box contains FIFO buffer, the received data can be stored in it. Through the synchronous detection institutions, we can ensure that any input training sequence can be used to initialize the receiver equalizer and synchronization signal.

After the module detects the signal synchronization, channel impulse can by estimated with channel estimation algorithm. Sync flag will be created at the same time, then notify the tap coefficient and coefficient calculation initialization module that which training sequence capture the synchronization signal. Then read estimated channel impulse response to initialize the equalizer.

To combat the channel estimation error that based on estimate algorithm, this paper adopts adaptive threshold control algorithm to filter out the noise interference and improve the channel estimation precision of the system. Then these estimated channel impulse will be used to initialize the taps of equalizer's feed-forward and feedback coefficient.

Considering the nature of the channel state, equalizer may not convergence after channel rapidly initialize, still unable to make the figure in the back of the eye open. To further accelerate the convergence speed of equalizer, against channel decay quickly, is proposed based on the output signal-to-noise ratio and symbol length threshold control method of loop multiplexing data. By the algorithm, can effectively use the training sequence from the transmission signal to improve the convergence speed of the equalizer.

\section{DESIGNED DFE EQUALIZER STRUCTURE BASED ON SPARSE ALGORITHM}

Compared with traditional decision feedback equalizer structure, the sparse tap initialize algorithm proposed in this paper just make changes tap in whether taps will be used in equalize calculate. Its structure as shown in figure2. 


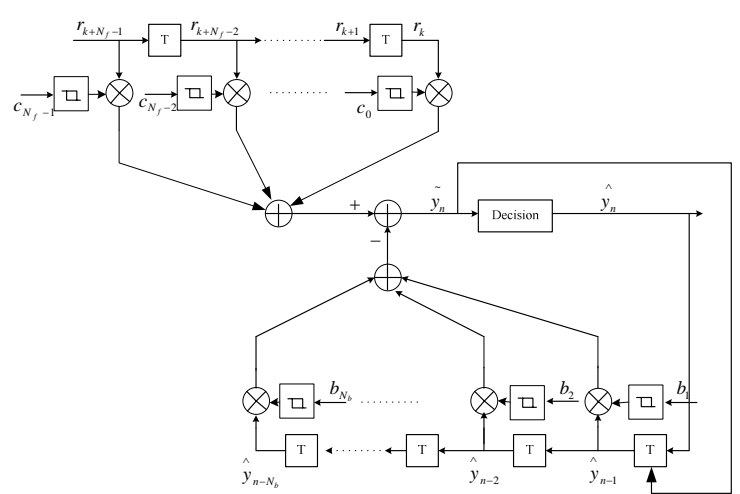

FIGURE III. PROPOSED DEF STRUCTURE BASED ON SPARSE ALGORITHM.

For this equalizer structure, we study which taps don't need to participate the calculation without reduce the equalize performance and reduce the excessive error [11]. Tap threshold calculation formula is given as follows.

$$
\left\{\begin{array}{l}
\operatorname{THF}(n)=\lambda_{F} \times\left(\sum_{q=1, q \neq n}^{N_{f}-1}\left|c_{q}\right|-\left|c_{n}\right|\right) /\left(N_{f}-1\right) \\
\operatorname{THB}(n)=\lambda_{B} \times\left(\sum_{p=1, p \neq n}^{N_{b}}\left|b_{p}\right|-\left|b_{n}\right|\right) /\left(N_{b}-1\right)
\end{array}\right.
$$

Among formula 3, $\operatorname{THF}(n)$ and $\operatorname{THB}(n)$ is the filter tap of feed-forward and feedback filter's corresponding threshold. $\lambda_{F}$ and $\lambda_{B}$ are used to control the tap coefficient threshold under this algorithm. In order to calculate conveniently, we use $\lambda_{F}$ and $\lambda_{B}$ as same $\lambda$. At the same time, in order to make the equalizer work stable, when the corresponding tap $w(j)$ is used to participate in automatic operation, the $\mathrm{L}$ a before and after the tap to be activated in tapping operations, no matter whether it has been activated. We set it as 15 here. The equalizer structure defined above was called SC-DFE.

In this algorithm, the $\lambda$ value direct related to excessive noise against performance. Here we analyzed the performance by simulation to determine which value make the system has best performance. Under the ITU walking mode set in the B channel, when the SNR is $20 \mathrm{~dB}$, the threshold level control method proposed in this paper, the different values of the simulation. The simulation results is shown in Figure 3.

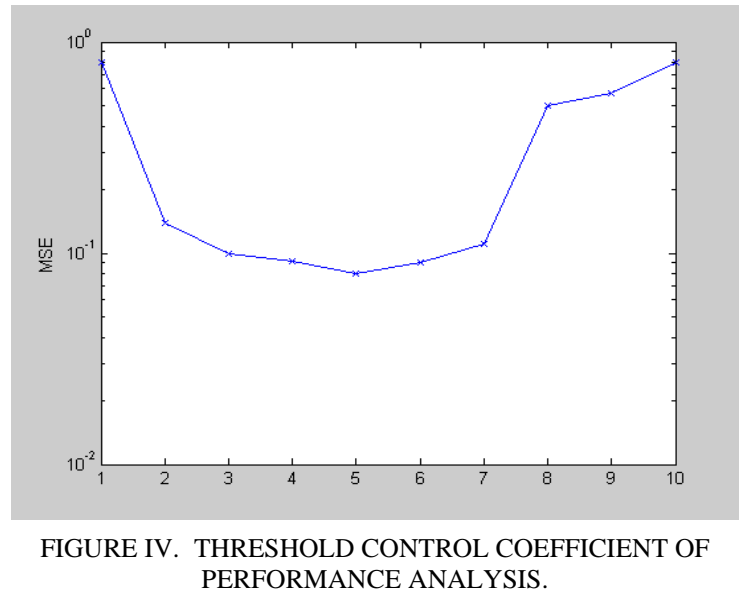

Through Figure 3 we can see, in the channel, the $\mathrm{D}$ value big than 7 will make equalizer work instability. And $D=5$ minimum the convergence mean square error so, we choose it as threshold control parameter.

\section{CONCLUSION}

On the results of previous studies, we make detailed and indepth study on channel estimation and equalization techniques based on ATSC-M/H protocol. Proposed a multi-channel training sequence framework. Considering the sparse multipath channel characteristics, proposed a sparse-tap equalizer channel estimation algorithm to eliminate the channel estimate noise. Provide a reference for the design of mobile digital television signal receive, also provides a theoretical basis for single-carrier modulation signal transmission system.

\section{ACKNOWLEDGEMENTS}

This work was supported by the Shanghai Second Polytechnic University Key Discipline Construction (4th term)Control Theory \& Control Engineering (No. XXKPY1308) and Shanghai Excellent Young Teachers Program (NO.B51YQ12R012).

\section{REFERENCES}

[1] ATSC, Guide to the use of the ATSC digital television standard. ATSC Doc.A/53, 1995.

[2] Y.Wu, S.Hirakawa, U.H.Reimers, Overview of digital television development world wide. Proc. IEEE, 2006, Vol.94 (1):8 21

[3] ETSI 300 421, Digital Video Broadcasting (DVB), Framing structure, channel coding and modulation for 11/12 GHz satellite services. 1997

[4] ETSI TR 102376 V1.1.1, Digital Video Broadcasting (DVB), User guidelines for the second generation system for Broadcasting, Interactive Services, News Gathering and other broadband satellite applications(DVB-S2). 2005

[5] ITU-R Recommendation M.1225, Guidelines for evaluation of radio transmission technologies for IMT-2000.1997

[6] Tests on Digital Television Systems. Third Report SET/ABERT, 2000

[7] ATSC, ATSC-mobile DTV standard, part 3 - service multiplex and transport subsystem characteristics. ATSC standard Document A/153 Part 3, 2009

[8] ATSC, ATSC Mobile DTV standard Document A/153 (A/153). 2011

[9] http://en.wikipedia.org/wiki/ATSC-M/H

[10] ATSC, Candidate Standard: ATSC-M/H Standard. 2009 
[11] N.I.Heo, D.S.Han, H.S.Oh, Adaptive Sparse Equalizer Robust to Fast Fading and Long Delay Spread for ATSC DTV. IEEE Transactions on Consumer Electronics, 2005, Vol.51 (3):803 808. 